Mediscope

The Journal of GMC

\title{
Pelvic congestion syndrome
}

\author{
R Sultana ${ }^{1}$, RA Begum², F Begum ${ }^{3}$
}

\begin{abstract}
Chronic pelvic pain is a common gynecological problem with many causes and may account for approximately $10 \%$ outpatient gynecological visit. Pelvic Congestion Syndrome (PCS) is defined as a condition characterized by congestion of the pelvic veins visible on selective ovarian venography in multiparous premenopausal women with a history of chronic pelvic pain for more than six months. We report a case of PCS in 35 years old multiparous lady complaining of chronic pelvic pain for one year. The pain was worsened by sitting and standing position. Other general symptoms were present such as dysmenorrhea, rectal discomfort and urinary frequency. On examination patient was depressed, there was abdominal and pelvic tenderness. Pelvic ultrasound and Doppler examination showed dilated and tortuous ovarian veins and dilated tortuous arcuate veins in the myometrium. An ovarian cyst was present and uterus was enlarged. In this case total abdominal hysterectomy was done although ideal treatment for PCS is ovarian vein embolisation by interventional radiology which is not available in our country.
\end{abstract}

Key words: Pelvic congestion syndrome, ovarian vein embolisation, pelvic varicosities, total abdominal hysterectomy

\section{Introduction}

Pelvic Congestion Syndrome (PCS) is defined as a condition characterized by congestion of the pelvic veins visible on selective venography in multiparous premenopausal women with history of chronic pelvic pain for more than six months. ${ }^{1}$ Chronic pelvic pain is a common gynecological problem with many causes and may account for approximately $10 \%$ of outpatient gynecological visits. The common causes of chronic pelvic pain include endometriosis, pelvic adhesions, atypical menstrual pain, urologic disorder, irritable bowel syndrome and psychological issues. Pelvic varicoceles are found in approximately half of women with chronic pelvic pain. ${ }^{1-4}$ Pelvic congestion is becoming increasing recognized as a cause of chronic pelvic pain. Hormonal factors contribute to vasodilatation and pelvic veins are exposed to high concentration of hormones produced

1. Dr. Razia Sultana MBBS, FCPS(Obs \& Gynae), Asso. Prof., Department of Obs \& Gynae, Gazi Medical College Khulna

2. Dr. Rowshan Ara Begum MBBS, FCPS(Obs \& Gynae), Prof., Department of Obs \& Gynae, Gazi Medical College Khulna

3. Dr. Ferdousi Begum MBBS, Sr. Medical Officer, Khulna Medical College Hospital, Khulna 
ovary. In a controlled study, women with pelvic congestion were noted to have ultrasound evidence of larger uterus and thicker endometrium. ${ }^{5}$ Incompetent ovarian valves are the causes of PCS in women which can cause chronic pelvic pain. Approximately, $15 \%$ of women experience pelvic congestion between the ages of 20 and 50 but not all experience symptoms. The symptoms are pelvic pain that worsens towards the end of day or after long period of standing, low back pain, vaginal discharge, continuous or recurring pain for at least 6 months, initial sensation of fullness or heaviness can increase during or after menstruation or after coitus. In addition $56 \%$ of women with pelvic congestion had cystic changes in their ovaries and menstrual disorders were frequently reported among them. Pelvic congestion occurs in the reproductive age group and suppression of ovarian activity results in reduction of pelvic congestion and pain. ${ }^{6}$

\section{The case}

Mrs. $X$ is 27 year old lady para one who presented with history of one year of left lower quadrant pain which was nagging pain, worse at night and often present late in the afternoon after prolonged periods of standing. She also complained with pain in groin region radiating to vulva, bladder irritation symptoms such as frequency and dysuria. The pain was noncyclical and refractory to analgesic therapy. In addition, she complained of dyspareunia and pain with bowel movements and her menstrual history was remarkable. Examination revealed no varicosities of lower extremity. Pervaginal examination revealed uterus bulky in size, fornices were tender, cervix was healthy and cervical excitation test was positive. Ultrasound examination demonstrated extensive pelvic varicosities. She was counseled for abdominal hysterectomy as there were no facilities for ovarian vein embolisation, the specific treatment of PCS. A total abdominal hysterectomy was performed. Intraoperatively, there were visible varicose veins through the peritoneum in the infundibulopelvic ligament. Ovaries were healthy and normal in size. Postoperative course was uneventful and she was discharged on $5^{\text {th }}$ postoperative day. She was asymptomatic in subsequent follow-up visits except pain in vulval and groin region due to vulval varicosities. Postoperative histopathological examination of uterus did not revealed any pathologic abnormalities. During discharge she was advised not to work in prolong standing position and pregabalin drugs were given for three months.

\section{Discussion}

The causes of ovarian varicoceles are probably multifactorial, involving both mechanical and hormonal factors. During pregnancy the vascular capacity of the ovarian veins may increase to 60 times the normal value and remain this way for months after delivery. ${ }^{7}$ Dilatation of ovarian veins causes vascular incompetence and retrograde venous flow. Dilated veins are more frequently present with increasing parity. ${ }^{8-11}$ The fact that PCS affects only premenopausal women suggests correlation between PCS and ovarian activity. ${ }^{10}$ Over times venous distension can render the valves incompetent. Additionaly, the weight gain with anatomic changes in the pelvic structures during pregnancy may cause chronic intermittent venous obstruction. Blood pooling in the pelvic and ovarian veins may cause further engorgement and thrombosis and mass effect on nearby nerves collectively contributing to pelvic pain. The complaint of chronic pelvic pain constitutes about $10-40 \%$ of all presentations to obstetrics and gynecology outpatient clinics. ${ }^{11-13}$ Pelvic ultrasound and computer tomography scans are usually the first imaging modalities in the evaluation of patients with chronic pelvic pain. Ultrasound with Doppler examination provides dynamic information about visualized venous blood flow. Criteria for the sonographic diagnosis of varices include: a) visualization of ovarian veins more than $4 \mathrm{~mm}$ in diameter, b) Dilated tortuous arcuate veins in the myometrium that communicate with bilateral pelvic 
varicose veins and c) slow blood flow and reverse cauda venous blood flow in the left ovarian vein.

Magnetic resonance imaging is the best primary imaging modality for this problem. Laparoscopy can still be negative in $80-90 \%$ of patients who do not have PCS as insufflations' of gas may cause compression of varices if present. Long term clinical studies have revealed coil embolization of patients with PCS has provided symptomatic relief in $74 \%$ of patients. ${ }^{4,12,13}$ Ovarian vein embolization relieved symptoms in $75 \%$ of patients with PCS in a study done by Park et al. ${ }^{11}$ Diagnosis of PCS is done by using transabdominal and transvaginal sonography. Women with PCS tend to have a large uterus and more cystic changes in ovaries than healthy women because of congestion and estrogen overstimulation. ${ }^{10}$ The diagnosis of chronic pelvic pain constitutes about $10-40 \%$ of all presentations to obstetrics and gynecology outpatient clinics. ${ }^{11-13}$ Pelvic pain among women is a common condition which may be caused by various reasons. The most common causes include endometriosis, pelvic adhesions, atypical menstrual pain, urological problems, pelvic varicocele, spastic colon syndrome and psychosomatic disorder. ${ }^{11}$ Dilatation of broad ligament and ovarian plexus veins and the presence of incompetent ovarian veins are a specific entity known as PCS or pelvic varicocele. Women with PCS are typically less than 45 years old and in their childbearing age. Ovarian veins increase in size related to previous pregnancies. Studies showed that $30 \%$ of patients with chronic pelvic pain have PCS as the sole cause of their pain and an additional 15\% have PCS along with another pelvic pathology.

\section{References}

1. Beard RW, Pearce S, Highmanm JH, Reginald PW. Diagnosis of pelvic varicosities in women with chronic pelvic pain. Lancet 1984;2:946-9.

2. Reiterre A. A profile of women with chronic pelvic pain. Clinic Obstet Gynecol 1990;33:130-6.
3. Giacchetto C, Cotroneo GB, Marincolo F, Cammisulif, Caruso G, Catizone F. Ovarian varicocele: ultrasonic and phebographic evaluation. J Clin Ultrasound 1990;18:551-5.

4. Cordis PR, Eclaves A, Buckley PJ, DeMarioribus CA, Cockerill ML, Yeager TD. Pelvic congestion syndrome: early clinical results after transcatheter ovarian vein embolisation. $J$ Vasc Surg 1998;28(5):862-8.

5. Hodson TJ, Reed MW, Peck RJ, Heningway AP. The Ultrasound and Doppler appearance of pelvic varices. Clin Radiol 1991;44:208-9.

6. Reginald PW, Adams J, Franks S, et al. Medroxy progesterone acetate in the treatment of pelvic pain due to venous congestion. $\mathrm{Br}$ J. Obstet Gynecol 1989;96:1148.

7. Giachetto C Catizone F, Cotroneo GB, et al. Radiologic anatomy of the genital venous system in female patients with varicocele. Surg Gynecol Obstet 1989;169:403-7.

8. Kennedy A, Hemingway A. Radiology of ovarian varices. $\mathrm{Br} J$ Hosp Med 1990;44:38-43.

9. Hoggkinson CP, Physiology of the ovarian vein during pregnancy Obstet Gynecol 1953;1:26-37.

10. Adams J, Reginald PW, Wadsworth JF, Beard RW. Uterine size and endometrium thickness and the significance of cystic ovaries in women with pelvic pain due to congestion. $\mathrm{Br} \mathrm{J}$ Obstet Gynecol 1990;97:583-7.

11. Park SJ, Lim JV, Ko YT, et al. Diagnosis of pelvic congestion syndrome using transabdominal transvaginal sonography. Am J Roentgenol 2004;182:683-8.

12. Belenky A, Bartal G, Atar E, Cohen M, Bachar GN. Ovarian varices in healthy female kidney donors: incidence, morbidity and clinical outcome. Am J Roentgenol 2002;179:625-7.

13. Harris RD, Hottzman SR, Poppe AM. Clinical outcome in female patients with pelvic pain and normal pelvis ultrasound findings. Radiology 